\title{
Résidus phytosanitaires dans les vins : état des lieux, analyses et expertise
}

\section{Phytosanitary residues in wines: Inventory, analysis and expertise}

\author{
M. Grinbaum ${ }^{1}$, M. Dubernet ${ }^{2}$, V. Bouazza ${ }^{2}$, E. Debez ${ }^{3}$, et V. Lempereur ${ }^{4}$ \\ ${ }^{1}$ Institut Français de la Vigne et du Vin, 2260 route du Grès, 84100 Orange, France \\ ${ }^{2}$ Laboratoires Dubernet, 35 rue de la Combe du Meunier, ZA du Castellas, 11100 Montredon des Corbières, France \\ ${ }^{3}$ Laboratoire Diœnos Rhône, 2260 route du Grès, 84100 Orange, France \\ ${ }^{4}$ Institut Français de la Vigne et du Vin, 210 Bd V. Vermorel CS 60320, 69661 Villefranche, France
}

\begin{abstract}
Résumé. Protéger la vigne à l'aide d'une substance active phytosanitaire, qu'elle soit d'origine de synthèse ou naturelle, peut entraîner la présence de résidus de cette substance ou de ses produits de dégradation dans le raisin puis dans le vin. Ces résidus sont réglementés et autorisés à condition qu'ils ne dépassent pas les limites maximales de résidus (LMR) exprimées en $\mathrm{mg} / \mathrm{kg}$. On voit cependant apparaître sur le marché de plus en plus de labels ou de chartes exigeant le «zéro résidu» dans les vins. Des précisions sont apportées pour faciliter l'interprétation d'une analyse de résidus et la nécessité d'une expertise technique pour accompagner ces résultats. Un état des lieux des résidus retrouvés dans les vins est ensuite dressé. Il confirme que les teneurs quantifiées sont en très forte diminution par rapport aux dernières décennies. Aucun dépassement de LMR n'est constaté, les concentrations moyennes étant inférieures à $1 \%$ des LMR. Enfin, un focus est réalisé sur deux molécules fongicides qui sont régulièrement retrouvées dans les vins, y compris dans les vins biologiques, pouvant entraîner des déclassements par les organismes certificateurs. Plusieurs hypothèses sur des sources d'apports autres que phytosanitaires en ces molécules dans les vins sont présentées.
\end{abstract}

Abstract. Protecting the vine with a phytosanitary active ingredient, whether synthetic or natural origin, may lead to the presence of residues of this substance or its products' degradation in grapes and wine. These residues are regulated and authorized provided that they do not exceed the maximum residue limits (MRLs) expressed in $\mathrm{mg} / \mathrm{kg}$. However, more and more labels or charters requiring «no residues » in wines appear on the market. Technical expertise is required to accompany the results and clarification is provided to facilitate the interpretation of a residue analysis. A residues' inventory found in the wines is made. It confirms that the quantified levels are in very strong decrease compared to the last decades. No MRLs were exceeded, with levels averaging less than $1 \%$ of the MRLs. Finally, a focus is made on two fungicides regularly found in wines, including organic wines, which can lead to their downgrading by certifying bodies. Several hypotheses, about sources of non-phytosanitary inputs into these two molecules in wines are presented.

\section{Introduction}

\subsection{Contexte}

Les produits phytosanitaires plus connus sous le terme de «pesticides» ont pour but de protéger les plantes contre les maladies en complétant leurs mécanismes naturels de défense, ils sont en quelque sorte «les médicaments des plantes ». La vigne est une plante qui fait l'objet de nombreuses agressions (champignons comme le botrytis, l'oïdium et le mildiou, mauvaises herbes et insectes parasites), lesquelles peuvent compromettre sa pérennité et la qualité des raisins et des vins.

La filière vitivinicole est par conséquent utilisatrice de produits phytosanitaires.

Actuellement, leur utilisation, leur présence dans l'environnement et les produits de consommation humaine, sont au centre des préoccupations des producteurs, des metteurs en marchés, et des consommateurs. Les viticulteurs sont de plus en plus nombreux à faire analyser leur vin afin de connaître précisément les quantités de résidus qu'ils contiennent.

L'objet de cet article est de présenter des résultats récents d'analyses de résidus dans les vins, de détailler la problématique de quelques molécules, et surtout de donner des éléments d'aide à l'interprétation d'un rapport d'analyse de résidus.

\subsection{Qu'est-ce qu'un résidu de pesticide ?}

Les produits phytosanitaires comprennent une gamme très étendue de produits composés de «substances actives» pouvant être classées dans différentes familles chimiques, comme les organochlorés, les organophosphorés, les carbamates ... Une fois appliqués sur la vigne, ces produits évoluent qualitativement et quantitativement au cours du temps. Le «résidu» est la quantité de 
substance active (ou de ses produits de dégradation) qui reste sur la partie consommable de la plante, au moment de la récolte.

L'essentiel des cultures est toujours soumis à des agresseurs naturels (insectes, champignons, bactéries...), qui peuvent compromettre tout ou partie d'une récolte, sur un plan quantitatif, qualitatif, voire sanitaire. La protection des cultures est un acte nécessaire et indispensable pour rendre possible une production agricole. Certains consommateurs semblent penser que «les résidus phytosanitaires ne sont pas autorisés dans les produits agricoles et donc dans les vins ». Or, à partir du moment où une protection est réalisée, à l'aide d'une substance active, qu'elle soit d'origine de synthèse ou naturelle, il est inévitable que l'on soit amené à retrouver des traces de celle-ci ou de ses produits de dégradation (résidus) dans les productions et dans l'environnement.

Ces résidus sont cependant très précisément réglementés et lorsqu'ils sont autorisés, c'est à condition qu'ils ne dépassent pas les limites maximales de résidus (LMR) exprimées en $\mathrm{mg} / \mathrm{kg}$.

Ces règlementations évoluent. Par exemple, en Europe, depuis une vingtaine d'années, le nombre de molécules autorisées a fortement diminué. Sur l'ensemble des 900 substances actives répertoriées en 1993 avant le début de l'évaluation européenne, plus de 70\% ont disparues. Dans un futur proche, l'interdiction des molécules classées CMR (cancérigène, mutagène, reprotoxique) ou perturbateurs endocriniens, devrait encore en limiter le nombre.

\subsection{Règlementation}

Avant leur utilisation par les viticulteurs, les produits phytosanitaires doivent être homologués. Cela signifie que la preuve de leur innocuité doit être apportée et que dans ce but, ils font l'objet d'une évaluation des risques au niveau français et européen. Rappelons qu'un produit phytosanitaire est un «produit formulé». Il correspond à la somme des substances actives (molécules à effet pesticide) et des adjuvants, qui améliorent leur efficacité (antioxydants, mouillants, émulsifiants, surfactants).

Ainsi, l'évaluation se fait à deux niveaux :

-la substance active : pour laquelle l'évaluation du risque pour l'homme et l'environnement, est réalisée dans le cadre européen, et a pour conséquence son approbation (ou non) pour une durée déterminée. -la spécialité commerciale : pour laquelle l'homologation se fait au niveau national. Une formulation ne peut être homologuée que si les substances actives qui la composent sont approuvées par le règlement européen.

C'est donc un processus long, comprenant de nombreuses études qui sont évaluées par des experts indépendants. Une molécule met de 10 à 15 ans avant d'être homologuée, ce processus est comparable à celui d'un médicament. En Europe, c'est le règlement (CE) $\mathrm{n}^{\circ} 1107 / 2009$ qui définit les règles de la mise sur le marché des produits phytopharmaceutiques. Celle-ci est ensuite subordonnée dans chaque pays à la détention d'une autorisation nationale publique.

Les Limites Maximales de Résidus de pesticides (LMR) autorisées dans les produits d'origine animale ou végétale et destinés à la consommation humaine sont fixées par le règlement européen (CE) $n^{\circ} 396 / 2005$. Elles ne sont définies que pour les matières premières (dans le cas du vin, les raisins de cuve) et non pour les produits transformés (le vin). Toutefois elles intègrent, dans leur calcul, les phénomènes de concentration ou de dilution inhérents à la vinification et le transfert des résidus du raisin au vin (facteurs de transfert). Ainsi, en l'état actuel de la réglementation européenne (non publication de l'annexe VI du règlement $(\mathrm{CE}) \mathrm{n}{ }^{\circ} 396 / 2005$ concernant la liste des facteurs de transfert spécifiques), un vin ne doit pas contenir des teneurs supérieures aux LMR fixées pour les raisins de cuve (avec un facteur de transfert de 1). A noter que pour les substances actives interdites ou les plus dangereuses, une LMR «par défaut» de $0.01 \mathrm{mg} / \mathrm{kg}$ est fixée.

\section{Analyse de résidus dans les vins}

\subsection{Matériels et méthodes}

Les natures chimiques des molécules phytosanitaires sont très diverses. Le récent large développement des analyses multi-résidus, dans des conditions technico-économiques acceptables a été rendu possible par les progrès des technologies et des méthodes de chromatographie gazeuse ou liquide couplée à la spectrométrie de masse en tandem (GC-MSMS et LC-MSMS).

La méthode OIV-MA-AS323-08, est la seule méthode normalisée officielle reconnue internationalement par la filière viti-vinicole. Cette méthode utilise une extraction par la méthode QuEchERS (Quick Easy Cheap Effective Rugged and Safe). Les extraits ainsi obtenus sont ensuite dosés par GC-MSMS ou par UPLC-MSMS.

Cette double méthode LC et GC multirésidus est complétée par deux autres méthodes qui ciblent des familles spécifiques de résidus :

- Méthode des dithiocarbamates, méthode publiée [1].

- Méthode QuPPe (fosétyl-Al, acide phosphoreux, Glyphosates et métabolites). Il s'agit d'une méthode normalisée européenne [2].

Les résultats présentés ici ont été obtenus avec les équipements suivants :

$$
\begin{aligned}
& \text { GC-MSM AGILENT } 6890 \text { - } 7000 \\
& \text { LC-MSMS WATERS Acquity TQD. }
\end{aligned}
$$

\subsection{Performances des méthodes}

Avant sa mise en service dans un laboratoire, une méthode d'analyse fait l'objet d'une validation. Cette dernière est définie par la norme ISO 17025/ 2017 comme une «vérification, où les exigences spécifiées sont adéquates pour un usage déterminé ».

Ainsi la validation est toujours associée à la définition de prérequis de performance, dont elle vérifiera la capacité de la méthode à les atteindre. Ces prérequis ont différentes origines : besoins des demandeurs, exigences normatives, règlementations (lignes directrices européennes...). Selon les contextes et les laboratoires, ces prérequis ne seront pas nécessairement définis de la même façon, et les validations ne concerneront donc pas nécessairement les mêmes niveaux de performance.

Il faut cependant souligner que, par le truchement d'essais interlaboratoires, de l'accréditation, et des demandes du marché mondialisé, les niveaux de performances entre laboratoires s'appuient sur des critères qui sont 
naturellement de plus en plus convergents. En Europe, le Guide SANTE/11813/2017 de la commission européenne, définit aussi un certain nombre de critères de bonnes pratiques et de performances exigées pour les laboratoires réalisant des analyses de résidus dans les aliments.

\subsubsection{Définitions des critères de performance}

Les définitions données en italique ci-dessous sont issues de la résolution OIV ENO 418/2013 [3].

Les performances d'une méthode d'analyse se définissent selon plusieurs critères, l'objectif étant que l'exactitude d'un résultat, «étroitesse de l'accord entre la valeur trouvée et la valeur vraie d'un mesurande», soit la plus faible possible, à l'intérieur des critères définis pour la validation.

L'erreur aléatoire est caractérisée en termes de fidélité de la méthode, c'est-à-dire «l'étroitesse de l'accord entre les valeurs mesurées obtenues par des mesurages répétés du même objet ou d'objets similaires dans des conditions spécifiées $\gg$.

L'erreur systématique se traduit en terme de biais de la méthode.

Les analyses de résidus dans les vins sont des analyses dites de «traces». C'est-à-dire qu'elles concernent des niveaux de concentrations qui se situent aux limites basses des méthodes. À ces limites basses, les notions de limite de quantification (LQ) et limite de détection (LD) constituent des critères de performance essentiels.

La limite de quantification est «la plus petite concentration pouvant être quantifiée avec une incertitude acceptable, dans les conditions acceptables décrites de la méthode. En l'absence d'exigences règlementaires ou normatives, l'incertitude acceptable sur la limite de quantification est fixée à $60 \%$ de la limite de quantification, par convention». Ceci signifie qu'un résultat donné à la limite de quantification est normalement associé à une erreur de mesure (incertitude) de $+/-60 \%$.

La limite de détection est «la valeur mesurée par une procédure de mesure donnée, pour laquelle la probabilité de déclarer faussement l'absence d'un constituant dans un matériau est $\beta$, étant donné la probabilité $\alpha$ de déclarer faussement sa présence ». En pratique, la limite de détection est conventionnellement estimée comme étant $1 / 3$ de la limite de quantification.

Les limites de quantification et de détection sont donc indissociables d'un résultat d'analyse de trace, afin de permettre son interprétation.

En outre, tout résultat d'analyse, doit pouvoir être observé en prenant en compte l'incertitude qui lui est associée. L'incertitude de mesure est «un paramètre non négatif qui caractérise la dispersion des valeurs attribuées à un mesurande, à partir des informations utilisées ». L'incertitude n'est donc pas un paramètre invariable associé à une méthode, comme cela est trop souvent compris. L'incertitude, ou l'erreur de mesure, est une variable, qui varie en fonction de la concentration, et qui est, le cas échéant, modélisable par une fonction, non linéaire de la concentration (10), et tend vers l'infini quand la concentration tend vers 0 (Fig. 1). Pour cette raison simple et fondamentale, le $\ll 0 »$ analytique n'est jamais atteignable. $« 0 »$ ne peut pas constituer un résultat analytique.
Erreur de mesure U(x) \% Dimétomorphe

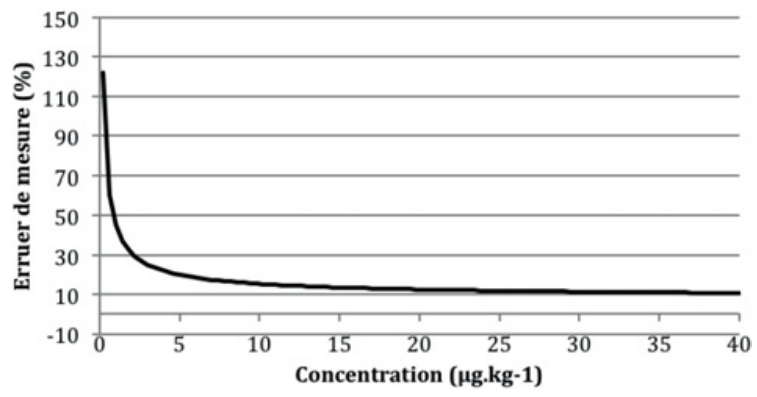

Figure 1. Modélisation de l'erreur de mesure en fonction de la concentration pour le dimétomorphe par LC-MSMS (source : Laboratoires Dubernet)

Selon la définition donnée dans la résolution OIV ENO 418/2013, l'incertitude est supposée atteindre + /$60 \%$ du résultat à la limite de quantification. Aussi, pour des analyses de résidus dans les vins, dont les teneurs sont généralement proches des limites de performance des méthodes, il est tout à fait normal et attendu que les laboratoires délivrent des incertitudes de l'ordre de $+/-20$ à $60 \%$ du résultat.

\subsubsection{Assurance qualité}

De tels niveaux d'incertitude sont tout à fait compatibles avec l'idée même de qualité et d'accréditation.

L'accréditation ISO 17025 assure qu'un laboratoire est audité régulièrement et qu'il travaille avec des méthodes validées, et selon les exigences techniques et organisationnelles de cette norme internationale. Elle constitue un véritable outil de reconnaissance, dans le monde entier, de la validité d'un résultat produit par un laboratoire. A cette reconnaissance de l'accréditation s'ajoute pour le vin et les moûts, le cas échéant, celle, tout à fait complémentaire de la mise en œuvre de la méthode normalisée OIV (OIV-MA-AS323-08).

Les exigences de l'accréditation conduisent les laboratoires accrédités à vérifier régulièrement leurs performances (qui peuvent évoluer dans le temps). Des outils de contrôle interne de la qualité, et des outils de contrôle externe, sont mis en œuvre. Parmi ces derniers, les essais interlaboratoires, qui permettent aux laboratoires de comparer leurs résultats, prennent une place privilégiée.

Selon les règles prévues dans la norme d'accréditation, les accréditeurs listent et publient les paramètres couverts par l'accréditation des laboratoires accrédités. Il est cependant tout à fait licite de rencontrer des rapports d'analyse faisant mention de l'accréditation, alors même que seule une partie des paramètres est couvert par la portée d'accréditation. C'est la situation la plus communément rencontrée. Plusieurs facteurs justifient cette accréditation partielle : les paramètres des résidus évoluent assez rapidement, et il est logistiquement difficile de faire coïncider les cycles d'audits avec ces évolutions. En outre pour certains paramètres il n'existe pas toujours des essais interlaboratoires permettant de faire des contrôles qualité externes attendus par les référentiels. 


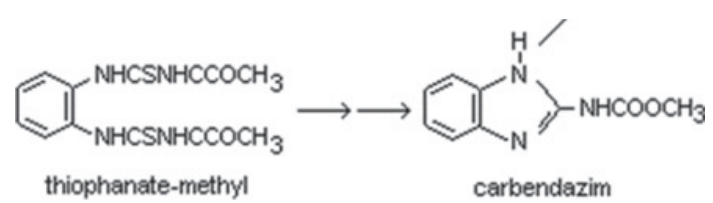

Figure 2. La carbendazime est un métabolite du thiophanate de méthyl.

\subsection{Interprétation d'un résultat}

\subsubsection{Principes généraux}

Les rapports d'analyses faisant mention de l'accréditation ISO 17025 répondent à des critères

Au-dessus de la limite de quantification, un résultat est rendu sous sa forme quantifiée. En Europe, et selon le Guide SANTE/11813/2017, l'unité à utiliser est le mg/kg.

En dessous de la $L Q$, un résultat sera rendu sous la forme $«\langle\mathrm{LQ} »$. Cela signifie que la concentration ne peut pas être quantifiée, mais un signal a été détecté.

En dessous de la LD, le résultat sera rendu sous la forme «nd» (non détecté).

Ainsi les valeurs de LQ et LD utilisées par les laboratoires sont des éléments à prendre en compte. Ces LQ et LD sont généralement très faibles au regard des LMR règlementaires. Cependant, lorsque l'utilisateur d'un résultat d'analyse recherche une présence / absence, les différences de LQ et LD entre laboratoires peuvent conduire à des interprétations différentes.

\subsubsection{Amélioration des performances analytiques et interprétation de résultats}

Les techniques ont beaucoup progressé ces dernières années, et continuent d'évoluer rapidement. Ces progrès sur les techniques GC-MSMS et LC-MSMS repoussent régulièrement les limites analytiques, en termes de justesse et de fidélité des mesurages, mais aussi en termes de limites de quantification et de détection. Ce qui n'était pas détectable il y a 10 ans, l'est devenu aujourd'hui.

Le « $0 »$ analytique n'existe pas, or à mesure de l'amélioration inexorable des performances des outils analytiques, on peut s'attendre à ce que des résultats d'analyse positifs basés sur des concentrations extrêmement faibles soient de plus en plus fréquents.

La limite interprétative de «résidus non détectés 》 est donc toute relative, et dépend, avant tout chose, des performances des méthodes utilisées.

$0.010 \mathrm{mg} / \mathrm{kg}$ est régulièrement employé en tant que limite de quantification «d'usage» pour les échanges commerciaux des produits conventionnels (codex alimentarius). Cette teneur constitue aussi la limite européenne «par défaut» fixée pour les molécules interdites. Elle est également retenue en tant que «valeur seuil» dans les produits biologiques par certains organismes certificateurs européens et dans la réglementation baby-food.

Cette teneur de $0.010 \mathrm{mg} / \mathrm{kg}$ fait donc régulièrement office de valeur de «minimis ». Une valeur de minimis étant une valeur analytique en dessous de laquelle une substance est consensuellement considérée comme absente du produit analysé.

Cependant, de fait, pour beaucoup de résidus les limites de quantification ont atteint ces dernières années des niveaux de concentration plus bas, régulièrement de l'ordre de 0.005 voire $0.001 \mathrm{mg} / \mathrm{kg}$.

En l'absence d'un cadre consensuel, normalisé et fort pour la définition de minimis, des débats complexes et parfois animés sur la présentation et l'interprétation des résultats continueront d'avoir lieu.

\subsubsection{Concentrations et nombre de molécules retrouvées}

Pour interpréter le résultat, il est également important de prendre en compte la provenance d'un vin.

Un vin issu d'un seul et même producteur aura tendance, le cas échéant, à présenter un spectre de résidus très limité en nombre de molécules.

En revanche, un vin issu d'assemblage provenant de vignobles et de producteurs différents, de pressions parasitaires différentes et donc de programmes de traitement très variés pourra présenter un spectre de molécules détectées plus large. Pour autant, certaines des teneurs quantifiées pourront s'avérer extrêmement faibles, en raison de l'effet de dilution dû à l'assemblage.

\subsubsection{Résidus et métabolites}

La définition analytique d'un résidu est, en Europe, donnée par la règlementation ( $\mathrm{R}(\mathrm{CE}) 559 / 2011)$.

Le plus souvent, un résidu est constitué de la seule molécule parent. Dans certains cas, il comprend la substance active elle-même ainsi que ses métabolites (ou produits de dégradation). C'est le cas par exemple du folpel, dont la définition du résidu correspond à la somme du folpel + phtalimide exprimé en folpel ou du fosetyl-al dont le résidu est la somme acide phosphoreux + fosetyl-al exprimée en fosetyl.

Dans le cas particulier des dithiocarbamates le résidu porte uniquement sur le CS2, produit de dégradation, commun à l'ensemble des molécules actives de cette famille.

Il arrive que le métabolite d'une molécule active soit une autre molécule active. C'est le cas notable du thiophanate méthyl, et de la carbendazime.

Le thiophanate-méthyl est une substance active autorisée en anti-botrytis sur vigne avec une LMR de $3 \mathrm{mg} / \mathrm{kg}$ dans le raisin de cuve.

La carbendazime était également une molécule active longtemps utilisée comme anti-botrytis sur vigne mais est interdite en Europe depuis 2009. Sa LMR devrait donc être de $0.01 \mathrm{mg} / \mathrm{kg}$. Or elle possède une LMR raisin de cuve de $0.5 \mathrm{mg} / \mathrm{kg}$ car elle est également un produit de dégradation du thiophanate de méthyl (Fig. 2). Il n'est donc pas illicite de rencontrer des résidus de carbendazime dans les vins, qui ne sont autres que des métabolites résultant de la dégradation du thiophanate-méthyl. Cette dernière molécule étant fréquemment utilisée, les probabilités de retrouver la carbendazim sont fortes. Toutefois, les teneurs en carbendazime dans les vins ne doivent pas dépasser la valeur de $0.5 \mathrm{mg} / \mathrm{kg}$.

\section{Etat des lieux résidus dans les vins}

Les résultats présentés ici sont issus de l'exploitation statistique des analyses en prestations réalisées entre 2015 et 2018 sur vins aux laboratoires Dubernet et Diœnos Rhône. 
Tableau 1. Moyennes des teneurs des 17 composés les plus fréquemment rencontrés dans les vins (hors phtalimide et acide phosphoreux).

\begin{tabular}{|c|c|c|c|}
\hline $\begin{array}{l}\text { Composé } \\
\text { Usage }\end{array}$ & $\begin{array}{l}\text { Proportion de vins avec des } \\
\text { teneurs détectées ( }>\text { LD) \% }\end{array}$ & Teneur moyenne (mg/kg) & $\begin{array}{c}\text { Moyenne exprimée en } \\
\text { \% LMR }\end{array}$ \\
\hline $\begin{array}{l}\text { Boscalid } \\
\text { Antifongique }\end{array}$ & $44 \%$ & 0.009 & $0.2 \%$ \\
\hline $\begin{array}{l}\text { Pyrimethanil } \\
\text { Antifongique }\end{array}$ & $29 \%$ & 0.015 & $0.3 \%$ \\
\hline $\begin{array}{l}\text { Dimethomorphe } \\
\text { Antifongique }\end{array}$ & $18 \%$ & 0.008 & $0.3 \%$ \\
\hline $\begin{array}{l}\text { Fenhexamid } \\
\text { Antifongique }\end{array}$ & $17 \%$ & 0.042 & $0.3 \%$ \\
\hline $\begin{array}{l}\text { Tebuconazole } \\
\text { Antifongique }\end{array}$ & $13 \%$ & 0.003 & $0.3 \%$ \\
\hline $\begin{array}{l}\text { Fluopyram } \\
\text { Antifongique }\end{array}$ & $13 \%$ & 0.006 & $0.4 \%$ \\
\hline $\begin{array}{l}\text { Métalaxyl } \\
\text { Antifongique }\end{array}$ & $11 \%$ & 0.005 & $0.5 \%$ \\
\hline $\begin{array}{l}\text { Fludioxonil } \\
\text { Antifongique }\end{array}$ & $10 \%$ & 0.008 & $0.2 \%$ \\
\hline $\begin{array}{l}\text { Iprodione } \\
\text { Antifongique }\end{array}$ & $9 \%$ & 0.028 & $0.3 \%$ \\
\hline $\begin{array}{l}\text { Fluopicolide } \\
\text { Antifongique }\end{array}$ & $9 \%$ & 0.004 & $0.2 \%$ \\
\hline $\begin{array}{l}\text { Iprovalicarbe } \\
\text { Antifongique }\end{array}$ & $9 \%$ & 0.008 & $0.4 \%$ \\
\hline $\begin{array}{l}\text { Ametoctradine } \\
\text { Antifongique }\end{array}$ & $7 \%$ & 0.004 & $0.1 \%$ \\
\hline $\begin{array}{l}\text { Benalaxyl } \\
\text { Antifongique }\end{array}$ & $7 \%$ & 0.001 & $0.3 \%$ \\
\hline $\begin{array}{l}\text { Cyprodinyl } \\
\text { Antifongique }\end{array}$ & $6 \%$ & 0.009 & $0.3 \%$ \\
\hline $\begin{array}{l}\text { Spiroxamine } \\
\text { Antifongique }\end{array}$ & $6 \%$ & 0.004 & $0.8 \%$ \\
\hline $\begin{array}{l}\text { Chlorantraniliprole } \\
\text { Antifongique }\end{array}$ & $3 \%$ & 0.008 & $0.8 \%$ \\
\hline $\begin{array}{l}\text { Carbendazime } \\
\text { Métabolite du } \\
\text { Thiophanante } \\
\text { méthyl } \\
\text { (Antifongique) }\end{array}$ & $3 \%$ & 0.011 & $0.4 \%$ \\
\hline
\end{tabular}

Des vins issus des vignobles européens de toutes origines et de toutes couleurs sont concernés. La base de donnée représente 4579 résultats. Une proportion non précisément connue des données est constituée de vins biologiques. Elle représente environ $15 \%$ des données.

Pour la totalité des échantillons, la méthode multirésidus GC-MS/MS et LC-MS/MS a été mise en œuvre.

Seule une partie de cette base de données (311 échantillons) a également fait l'objet d'une recherche par méthode QuPPe pour l'analyse, notamment de l'acide phosphoreux.

\subsection{Protocole}

\subsubsection{Vins analysés}

Les analyses portent sur des vins de millésimes différents (2012 à 2017).

Les calendriers de traitement ne sont pas connus, donc nous ne savons pas quelle molécule a été appliquée à la vigne.

\subsubsection{Molécules analysées}

140 molécules ont été analysées, représentant la très grande majorité des risques potentiels de résidus dans les vins. Certaines molécules sont exclusivement autorisées sur les cultures maraîchères ou fruitières ce qui permet de constater d'éventuelles contaminations (cas de vergers proches de parcelles de vignes).

Ainsi, 26 molécules utilisées pour le désherbage, 70 molécules fongicides et 44 molécules insecticides ont été recherchées dans nos échantillons, $38 \%$ d'entreelles ne sont pas autorisées sur vignes ou dans l'Union Européenne.

\subsection{Résultats}

\subsubsection{Fréquences des résidus dans les vins}

$32 \%$ des vins analysés ne présentent pas de résidus détectés et $75 \%$ présentent moins de 5 résidus différents détectés (Fig. 3).

Considérant la nature de la base de donnée, il apparaît qu'une population non négligeable de vins non bio, ne présente donc pas de résidus détectables. 
Proportion de vins par nombre de résidus détectés (>LD)

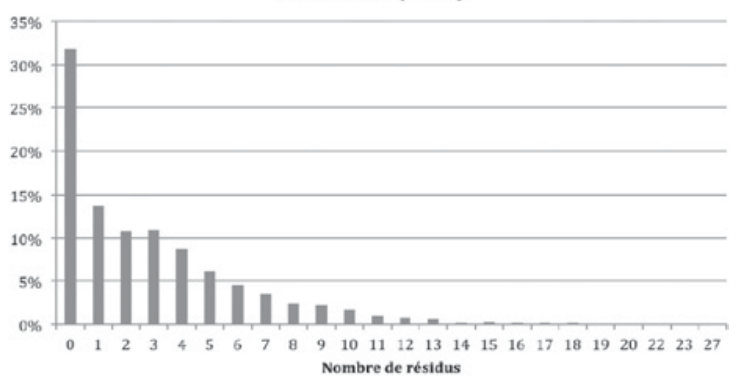

Figure 3. Proportion des vins en fonction du nombre de résidus détectés.

Tableau 2. Evolution des teneurs moyennes en $\mathrm{mg} / \mathrm{L}$ dans les vins depuis 2000 pour quelques molécules.

\begin{tabular}{ccccc}
\hline usage & molécules & 2000 & 2010 & 2018 \\
\hline botrytis & cyprodinil & 0.020 & 0.016 & 0.009 \\
\hline mildiou & dimétomorphe & 0.060 & 0.021 & 0.008 \\
\hline botrytis & fenhexamid & 0.125 & 0.060 & 0.042 \\
\hline mildiou & iprovalicarb & 0.050 & 0.027 & 0.008 \\
\hline botrytis & iprodione & 0.160 & 0.148 & 0.028 \\
\hline botrytis & pyrimethanil & 0.135 & 0.105 & 0.015 \\
\hline
\end{tabular}

\subsubsection{Teneurs rencontrées dans les vins}

Sur l'ensemble des vins analysés, aucun ne dépasse les LMR. Tous sont donc conformes à la réglementation européenne. Les teneurs moyennes détectées sont très faibles et constituent des fractions inférieures à $1 \%$ des LMR (Tableau 1).

Les molécules les plus fréquemment retrouvées sont toutes des antifongiques. Aucun insecticide, ni aucun herbicide n'a été détecté dans un vin.

A noter que sur les 140 molécules analysées, une dizaine seulement est responsable de l'immense majorité des résidus détectés dans les vins. Autrement dit, un grand nombre de produits phytosanitaires utilisés sur la vigne ne semble pas conduire à une présence détectable de résidu dans les vins, dans les conditions pratiques de leur emploi.

\subsection{3. Évolutions des teneurs mesurées dans le temps}

Les données actuelles sont confrontées aux données du plan de surveillance national mené par la DGAL dans les années 2000 [4] et à celles du plan de surveillance national mené par la filière vin en 2010 (non publiées).

De façon générale, on constate que les teneurs en résidus retrouvées dans les vins sont en très forte diminution par rapport aux dernières décennies (Tableau 2).

\section{Cas particuliers}

La présence dans les vins, de substances actives pourtant jamais utilisées dans l'exploitation peut être expliquée par une pollution environnementale ou une contamination croisée mais dans ces cas les teneurs retrouvées dans les vins sont souvent inférieures à $0.010 \mathrm{mg} / \mathrm{l}$.

Toutefois pour quelques molécules il existe un «bruit de fond de pollution» dans les vins qui peut atteindre

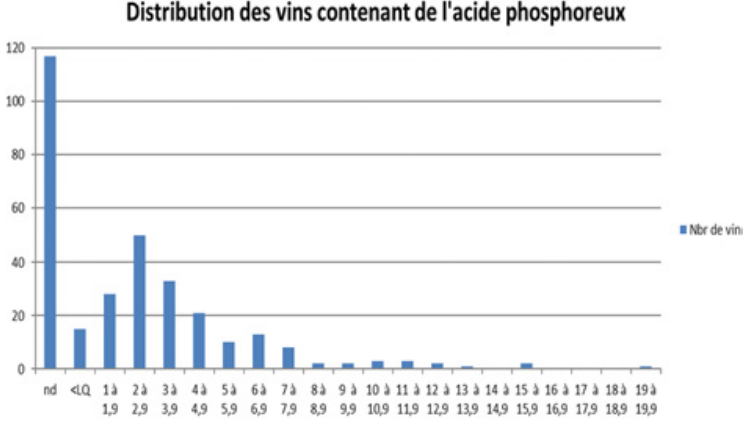

Figure 4. Nombre de vin contenant de l'acide phosphoreux (311 vins analysés) à des teneurs allant de 1 à $20 \mathrm{mg} / \mathrm{L}$.

des proportions plus importantes et poser des problèmes, notamment pour les vins biologiques [5].

C'est le cas de l'acide phosphoreux, produit de dégradation des phosphonates très utilisés en vigne sur le mildiou, comme fosetyl-al, disodium phosphonate, ou phosphonate de potassium, ainsi que du phtalimide, métabolite du folpel.

Un résidu est règlementairement défini par une molécule, ainsi que ses éventuels métabolites. Cette conception implique que les métabolites produits soient issus avec certitude de la dégradation de la molécule mère telle que donnée dans la définition règlementaire.

Il apparaît que dans ces deux cas de l'acide phosphoreux et du phtalimide, l'hypothèse de l'exclusivité de leur origine phytosanitaire, telle que prévue par la réglementation, ne soit pas vérifiée.

\subsection{Acide phosphoreux}

\subsubsection{Présence dans les vins}

Dans la plante, le fosetyl d'aluminium (sel d'aluminium de l'acide ethyl phosphoreux) se dégrade très rapidement en acide phosphoreux ou acide phosphonique (H3PO3). La LMR européenne en vigueur dans les raisins porte sur la somme fosetyl-al et acide phosphoreux exprimée en fosetyl. Elle est de $100 \mathrm{mg} / \mathrm{kg}$ [6]. Or, l"acide phosphoreux est retrouvé très fréquemment dans les vins $[7,8]$.

Dans notre étude, 311 vins ont été analysés et son taux de fréquence de détection est de l'ordre de $90 \%$. Les teneurs retrouvées varient de 0.1 à $20 \mathrm{mg} / \mathrm{l}$ avec une moyenne de $4.2 \mathrm{mg} / \mathrm{L}$ (Fig. 4).

En revanche, le fosetyl se retrouve très rarement dans les vins à la limite de quantification $0.5 \mathrm{mg} / \mathrm{l}$.

L'application de phosphonate quels qu'ils soient, entraîne la présence d'acide phosphoreux dans les vins, les teneurs étant d'autant plus élevées que la quantité de substance active cumulée apportée à l'hectare est importante. Toutefois, il n'est pas possible d'établir une corrélation entre la teneur en acide phosphoreux dans les vins et l'application de phosphites à la vigne.

Des tendances peuvent être observées : teneurs plus élevés si nombre de traitement avec l'ensemble des phosphites (fosetyl, disodium phosphonate et phosphonate de K) est élevé et que le dernier traitement est tardif. Les teneurs maximales (jusqu'à $50 \mathrm{mg} / \mathrm{L}$ mesurés) sont observées dans des parcelles ayant reçu le plus grand nombre d'applications (6 à $1800 \mathrm{~g} / \mathrm{ha}$ soit plus de $10000 \mathrm{~g} / \mathrm{ha} / \mathrm{an}$ cumulés). Mais cela n'est pas toujours vérifié $[7,9]$. 
De plus, des teneurs significatives en acide phosphoreux sont retrouvées sur des vins dont il a pu être établi avec certitude l'absence de traitements utilisant des phosphonates.

Ainsi, l'acide phosphoreux est régulièrement retrouvé dans les vins bios, pouvant entraîner des déclassements de lots par les organismes de certification.

Un «bruit de fond» est constaté de l'ordre de 0,1 à $3 \mathrm{mg} / \mathrm{L}$ d'acide phosphoreux dans des vins qui n'ont pas été traités avec des phosphites [5].

Dans les vins issus de raisins traités avec des phosphites, les teneurs sont généralement observées au-dessus de $3 \mathrm{mg} / \mathrm{L}$ [9].

\subsubsection{Hypothèses de sources d"apport en acide phosphoreux dans les vins non traités}

Plusieurs sources d'apports qu'elles soient d'origine phytosanitaire ou non, sont suspectées :

- Origine endogène.

- Application d'engrais phosphatés, produits fertilisants ou autres produits à base de phosphore utilisés en bio,

- Application d'amendements ou engrais organiques issus du traitement des sous produits viti-vinicoles conventionnels (compost de marc et de lies par exemple), utilisés en bio,

- Accumulation dans la plante et dans les sols due aux traitements des années antérieures,

- Dérive des traitements des parcelles voisines conventionnelles,

- Utilisation de sels de phosphate diammonique (DAP) pour la nutrition des levures en fermentation. Des premiers essais pour vérifier cette hypothèse ont été réalisés par nos soins. Aucune production d'acide phosphoreux n'a pu être établie.

Des recherches menées par l'Institut Français de la Vigne et du Vin sont en cours pour vérifier chacune de ces hypothèses.

\subsubsection{Seuil proposé}

Compte-tenu du «bruit de fond» observé avec cette molécule dans les vins issus de raisins non traités, il n'est pas envisageable de conclure à une présence de résidus phytosanitaires sur des vins qui contiendraient des teneurs en acide phosphoreux inférieures au seuil de $3 \mathrm{mg} / \mathrm{L}$.

\subsection{Phtalimide}

\subsubsection{Présence dans les vins}

Le folpel se dégrade en phtalimide $(80 \%)$ puis en acide phtalamique et enfin en acide phtalique.

La LMR raisin de cuve folpel a été modifiée suite au règlement 2016/156 de la commission du 18/01/16 modifiant les annexes II et III du règlement $\mathrm{CE}$ $n^{\circ} 296 / 2005$. Elle prend désormais en compte le phtalimide dans la définition du résidu et est de $20 \mathrm{mg} / \mathrm{kg}$ (somme folpel + phtalimide exprimée en folpel).

Le folpel peut se retrouver dans les raisins et les moûts mais n'est généralement plus présent dans les vins car il s'hydrolyse rapidement en milieu aqueux en phtalimide. Ce dernier, en revanche, est régulièrement retrouvé dans

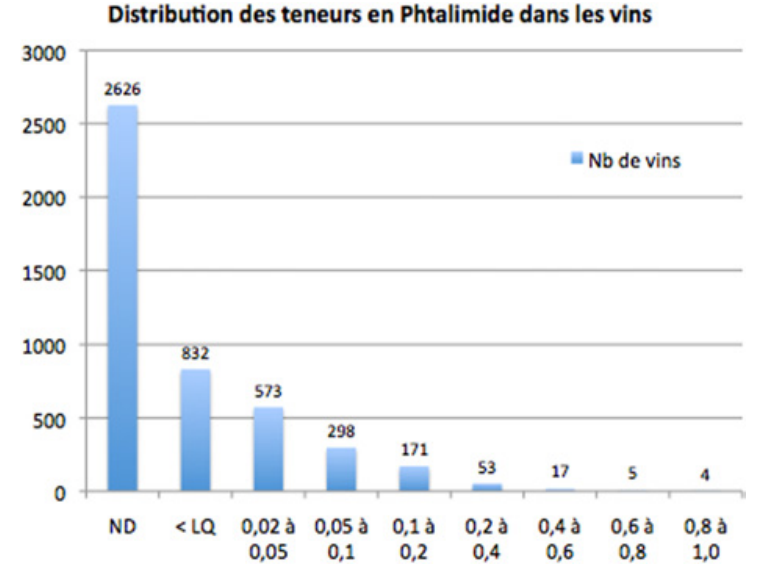

Figure 5. Distribution des teneurs en phtalimide dans les vins (4579 analyses- teneurs allant de 0.02 à $0.95 \mathrm{mg} / \mathrm{L}$ ).

les vins. Cette molécule est assez stable dans le vin car six mois après la vinification, les résidus de phtalimide restent inchangés.

Dans notre étude, le phtalimide est, avec l'acide phosphoreux, l'élément le plus fréquemment retrouvé sur les analyses de résidus. Il est détecté dans $43 \%$ des vins sur la base de données de 4579 échantillons. Il est quantifié dans $25 \%$ des vins à une teneur moyenne de $0.047 \mathrm{mg} / \mathrm{L}$ et une teneur maximale, atteinte dans de rares cas, de $0.95 \mathrm{mg} / \mathrm{L}$ (Fig. 5).

\subsubsection{Hypothèses de sources d"apport en phtalimide dans les vins non traités}

D'un point de vue réglementaire, le phtalimide est considéré comme un marqueur de la dégradation du Folpel, utilisé comme fongicide [10,11]. Toutefois, si l'utilisation de folpel conduit à la présence de phtalimide, la réciproque n'est pas vérifiée.

En effet, cette molécule est régulièrement retrouvée dans des vins issus de raisins n'ayant fait l'objet d'aucun traitement contenant du folpel, y compris des vins bios. Pour ces derniers, la présence non justifiée de phtalimide entraîne des difficultés pouvant aller jusqu'au déclassement du lot par les organismes de certification.

Le «bruit de fond» constaté dans les vins issus de raisins qui n'ont pas été traités avec le folpel est généralement de l'ordre de 0.010 à $0.040 \mathrm{mg} / \mathrm{L}$ [5].

Dans les vins issus de raisins traités au folpel, les teneurs évoluent généralement au-dessus de 0.03 à $0.05 \mathrm{mg} / \mathrm{L}[8,9,12]$.

L'hypothèse d'un apport autre que phytosanitaire en phtalimide dans les vins n'est pas à écarter car c'est une molécule qui sert aussi à fabriquer du caoutchouc ou stabiliser du polychlorure de vinyle (PVC) Les dérivés de phtalimide peuvent également être utilisés comme agents de polymérisation de certaines résines époxy. La mise en contact des vins avec des matériaux plastiques utilisés en vinification (tuyaux, tapis à vendange, résines époxy) est ainsi suspectée d'être une des causes de la presence de cette molecule dans le vin.

Une autre hypothèse a été proposée par une étude récente menée par un laboratoire membre de Relana (Labor FriedleGmbH). Elle tend à montrer que les faibles teneurs de phthalimide retrouvées, seraient un artéfact formé lors de l'analyse elle-même par GC-MS/MS. Une 
réaction de l'anhydride phtalique (PSA) avec des amines contenues dans la matrice alimentaire à haute température, en serait à l'origine $[13,14]$. Les premiers essais pour reproduire cette hypothèse dans nos conditions n'ont pas abouti à une production de phtalimide. D'autres essais sont en cours.

Des recherches menées par l'Institut Français de la Vigne et du Vin sont en cours pour vérifier ces hypothèses.

\subsubsection{Seuil proposé}

Compte-tenu du «bruit de fond» observé avec cette molécule dans les vins issus de raisins non traités, il n'est pas envisageable de conclure à une présence de résidus phytosanitaires sur des vins qui contiendraient des teneurs en phtalimide inférieures au seuil de $0.04 \mathrm{mg} / \mathrm{L}$.

\subsection{Conclusion}

L'intégration de l'acide phosphoreux, ou du phtalimide, dans la définition règlementaire des résidus est basée sur l'exclusivité phytosanitaire de leur origine. Or tout montre que ces hypothèses ne sont pas exactes.

Il en découle aujourd'hui tout une série de conséquences néfastes sur l'interprétation et l'usage qui est fait des analyses de résidus dans les vins.

En outre, la considération toxicologique de la présence d'acide phosphoreux ou de phtalimide, est identifiée à celle des molécules phytosanitaires dont ils sont supposés issus (fosétyl-Al, et folpel, respectivement). Or leurs toxicités ne sont pas du tout du même ordre. Le phtalimide est en effet, considéré comme non toxique, car il est excrété rapidement par l'organisme $[10,15]$. Quand à l'acide phosphoreux il présente également une toxicité faible [16].

\section{Discussions, perspectives}

L'analyse de résidus se démocratise au sein du monde viti-vinicole, en raison des demandes sociétales, du développement de la viticulture biologique, et du souhait des producteurs conventionnels de mieux maîtriser cette question afin de s'améliorer.

L'analyse de résidu relève de paramètres extrêmement complexes : méthodes d'analyse, réglementation, interprétation, signification. Une expertise technique est requise pour accompagner un résultat, et interpréter le rapport d'analyse.
La question des résidus de pesticides dans le vin est un sujet devenu sociétal, et donc très sensible car il ne relève pas seulement de données objectives et scientifiques. C'est un fait qui ne peut être ignoré, et doit être pris en compte.

Cette étude montre la présence de résidus dans certains vins, mais les concentrations moyennes retrouvées dans les vins que nous avons analysés sont toujours très en dessous des LMR raisin de cuve $(<1 \%$ des LMR).

Les teneurs relevées aujourd'hui seraient pour près de la moitié, passées inaperçues il y a 10 ans, en raison de l'amélioration des performances analytiques.

La totalité des vins est conforme à la réglementation.

Les données montrent que la situation continue encore à s'améliorer car les teneurs en résidus dans les vins sont toujours plus faibles.

La prise en considération de la gestion des résidus phytosanitaires dans les vins par les professionnels de la filière est devenue incontournable, non seulement pour les vins bios, mais aussi pour les vins issus de l'agriculture conventionnelle.

\section{Références}

[1] H.B. Cesnik, A. Gregorcic, Acta Chim. Slov. 53, 100 (2006)

[2] EU Ref. Lab. For Residues of Pest. (2013)

[3] OIV resolution ENO 418/2013 (2013)

[4] J.P. Cugier, S. Bruchet, Plan de surveillance DGAL (2005)

[5] S. Becquet, SECURBIO (2014)

[6] E. Malusa, L. Tosi, Food Add. Cont. 22, 541 (2005)

[7] M. Grinbaum, ITAB Avignon (2012)

[8] M. Grinbaum, AREDVI (2015)

[9] M. Grinbaum, RDOE 164 (2017)

[10] P. Cabras, A. Angioni, V.L. Garau, J.O.A.F.C 45, 476 (1997)

[11] A. Viviani-Nauer, P. Hoffmann-Boller, J. Gafner, Am. Jo. En. Vi. 4867 (1997)

[12] M. Dubernet, V. Bouazza, P. Duprat, M. Toussaint, E. Debez. RFOE 9 (2015)

[13] Relana Position Paper $n^{\circ} 16$ (2016)

[14] Relana Position Paper n ${ }^{\circ} 17$ (2017)

[15] EFSA Scientific report 297, 14 (2009)

[16] EFSA Scientific report 54, 1 (2005) 